\section{Bipolar disorder and disruptive mood dysregulation in children and adolescents: assessment, diagnosis and treatment}

\author{
doi:10.1136/eb-2013-101400
}

Bipolar disorder $(\mathrm{BD})$ is a heritable psychiatric condition, that is, associated with lifelong distress and impairment. There has been a long-standing interest in the early-life origins of BD, not least because as many as $50 \%$ of adults with $\mathrm{BD}$ report onset of their symptoms during childhood and adolescence. ${ }^{1}$ In this article, we present evidence on the assessment and treatment of $\mathrm{BD}$ and related conditions in young people. We also discuss the recent controversy about $\mathrm{BD}$ in children and adolescents that has led to the introduction of the diagnosis of disruptive mood dysregulation disorder (DMDD) by the Diagnostic and Statistical Manual for Mental Disorders (DSM)-5.

\section{MANIA}

\section{Diagnostic features}

$\mathrm{BD}$ is characterised by episodes, that is, distinct periods of abnormally and persistently elevated, expansive or irritable mood. During these episodes, patients experience symptoms such as grandiosity, distractibility, decreased need for sleep, pressure of speech, an increase in activity, flight of ideas and psychomotor agitation. These episodes are required to last by at least 4 days to diagnose hypomania or at least 7 days to diagnose mania. The insistence on episodic mood changes is crucial and prevents clinicians from rating symptoms such as the chronic concentration problems of a child with attention deficit hyperactivity disorder (ADHD) as evidence for mania. ${ }^{2}$ Manic episodes are extremely rare in children and relatively rare in adolescents according to epidemiological studies in the UK and the USA. ${ }^{3}$

\section{Acute treatment}

Pharmacotherapy is the mainstay of treatment for acute mania. Aripiprazole, quetiapine and risperidone are approved by the US Food and Drug Administration (FDA) for the treatment of mania in children and adolescents, and olanzapine in approved only for adolescents. A comparative analysis of randomised, placebo-controlled trials in youth BD established that atypical antipsychotics have better efficacy and tolerability compared with anticonvulsants and lithium (effect size atypical antipsychotics 0.65 , CI 0.53 to 0.78 vs anticonvulsants and lithium $0.24,95 \%$ CI 0.06 to 0.41 ). Particularly in youth, atypical antipsychotics caused significantly more weight gain than anticonvulsants (effect size atypical antipsychotics 0.53 , CI 0.41 to 0.66 vs anticonvulsants 0.10 , CI 0.12 to 0.33$){ }^{4}$

\section{Maintenance treatment}

The goal of maintenance treatment is mood stabilisation; keeping symptom improvements of acute treatment, and preventing recurrence of episodes. Assessment of residual symptoms, treatment adherence and side effects are part of relapse prevention. The only long-term trial in youth with $\mathrm{BD}$ is a 30 -week, randomised controlled trial showing that aripiprazole was superior to placebo in improving mania symptoms at endpoint. ${ }^{5}$ However, completion rates were low (32.4\%), limiting the inferences that can be drawn about it. We need more evidence of maintenance treatment in young people with BD.
This is particularly important because of the potentially serious long-term side effects of antipsychotic medication.

Adjunctive psychosocial and familial interventions might play an important role in relapse prevention of youth $\mathrm{BD}$. Family-focused treatment (FFT) is a psychoeducational intervention designed to reduce familial stress, conflict and affective arousal by enhancing communication and problem-solving among patients and caregivers, and it appears to ameliorate the course of BD in adolescents. ${ }^{6}$

\section{BIPOLAR DEPRESSION}

Many young people with $\mathrm{BD}$ will present with symptoms of depression before experiencing a full-blown manic episode and may spend more time of their illness being depressed than manic. It appears that youth with bipolar depression have more severe depressive episodes, associated with suicidality, hopelessness, and anhedonia compared with youth with unipolar depression, ${ }^{7}$ although further studies are required to distinguish between the two.

\section{Psychological treatment}

Preliminary results on cognitive behavioural therapy (CBT) for bipolar depression have shown a significant decrease in depressive symptoms. Given the evidence for effectiveness of CBT in unipolar depression, it seems reasonable to offer CBT for BD depression. The use of CBT adjunctive to pharmacotherapy or alone should rely on intensity of symptoms, level of impairment and cognitive resources of the patient. ${ }^{7}$

FFT has also shown promising results. In a randomised trial comparing effectiveness of FFT with enhanced care (only psychoeducation) in addition to pharmacotherapy in adolescents, the FFT group recovered faster from depressive symptoms, spent fewer weeks in depressive episodes and had a more favourable trajectory of depression symptoms for 2 years. ${ }^{6}$

\section{Pharmacological treatment}

There is no FDA-approved medication for bipolar depression in children and adolescents. Medications used to treat bipolar depression in adults are commonly used in the treatment of children and adolescents. Quetiapine is an effective treatment of depressive episodes in adults with $\mathrm{BD}$, and it seems reasonable to use this medication in older adolescents with BD.

\section{SHORT-LIVED MANIC EPISODES}

The duration requirement-a minimum of 7 days for mania or 4 days for hypomania is arbitrary and has been challenged in the literature about adults with $\mathrm{BD} .^{8}$ Moreover, it has been argued that shorter episodes may be more characteristic of mania in children. Episodes of shorter duration (between 1 and 3 days) during which young people experience manic symptoms are certainly much more common than classical (hypo-) mania in general population samples. ${ }^{3}$ In addition, evidence from longitudinal clinical studies suggests that up to $40 \%$ of people who experience such shorter episodes (often termed $\mathrm{BD}$-not otherwise specified, BD-NOS) may go on to develop classical (4-7 days) BD. ${ }^{9}$ However, more evidence is required to decide whether these short-lived episodes of mania-like symptoms are aetiologically linked with $\mathrm{BD}$.

\section{Treatment}

There is no trial evidence available for the treatment of such episodes. These episodes, which can often cause substantial impairment, can present a challenge to clinicians. We monitor such patients closely using weekly mood monitoring and offer several 
sessions of psychoeducation to them and their family where we explain the relative merits of available treatment options. In our own experience, treatment of comorbid conditions (eg, ADHD) and family-based interventions or CBT can help reduce the frequency of such episodes. Typically, we reserve treatment with antimanic agents only for those who fall short of the duration requirement by a small margin, for example, children with clear manic symptoms lasting for 3 days, or those who have been resistant to other treatment options. ${ }^{10}$

\section{BIPOLAR CONTROVERSY: IRRITABILITY AND DISRUPTIVE MOOD DYSREGULATION DISORDER}

Over the last 20 years, there has been a dramatic - up to $500 \%$ increase in the diagnosis of BD in children and adolescents in the USA. ${ }^{2}$ At the same time, there was a steep rise in antipsychotic prescriptions in the USA, ${ }^{11}$ raising concerns that children were overdiagnosed and unduly prescribed potentially harmful medication. The observed increase is unlikely due to a genuine increase in the prevalence of the disorder, but rather reflect changes in diagnostic practice. In particular, it has been argued ${ }^{2}$ that children presenting with severe irritability without clearcut episodes have been mislabelled as suffering from BD. Such chronic irritability needs to be contrasted to episodic mood change (either elation or irritability or depression, as discussed above) that lasts several days to a few weeks and is typical of bipolar disorder. To test the claim that children with severe chronic irritability had an early form of $\mathrm{BD}$, Leibenluft et al created a new category entitled Severe Mood Dysregulation $(\mathrm{SMD})$, that has chronic negative mood in the form of irritability and frequent temper outbursts as its symptoms. Several studies have shown that chronic and episodic irritability follow distinct trajectories; episodic symptoms are associated with mania, whereas chronic symptoms are strongly associated with unipolar depression and anxiety. ${ }^{12}$ Longitudinally, episodic irritability in early adolescence was associated with generalised anxiety disorder (GAD) and mania in late adolescence, and only mania in adulthood, whereas chronic irritability in early adolescence was associated with disruptive behavioural disorders in late adolescence and only major depressive disorder (MDD) in adulthood. ${ }^{13}$ Individuals with episodic and elated mood are up to 50 times more likely to develop mania than those with chronic irritability in a 3-year follow-up. ${ }^{14}$

On the basis of the research on SMD, and the importance of chronic irritability for developmental psychiatry, the DSM-5 working group proposed a new diagnostic category called DMDD. The definition of this new category resembles the one for SMD (severe recurrent temper outbursts superimposed on persistently irritable or angry mood); however, DMDD does not include the hyperarousal requirement of SMD. There is still little evidence on DMDD itself so far, although the announcement by the DSM has led to concerns that normative children's behaviours (tantrums) would be pathologised. Recently Copeland et al, ${ }^{15}$ using two community samples, suggested that DMDD was relatively rare, ranging from $0.8 \%$ to $3.3 \%$, with higher rates in preschool age and in boys. In the same study, DMDD significantly cooccurred with all common psychiatric disorders particularly with depressive disorders (OR between 9.9 and 23.5) and oppositional defiant disorder (OR between 52.9 and 103.0).

It remains unclear whether severe irritability should be declared a disorder or seen as the extreme of a spectrum. ${ }^{16}$ Empirical evidence supports chronic irritability as a continuum; however, clinical decisions require thresholds and binary disease categories are commonly used for such purposes in many other disorders.

\section{Treatment}

With regard to pharmacological options for the treatment chronic irritability, the only randomised controlled trial in children with SMD found no benefit of lithium over placebo. An open-label trial using low doses (mean $1.2 \pm 0.5 \mathrm{mg}$ ) of risperidone in children and adolescents with SMD showed significant reductions in irritability scores. ${ }^{17}$

Parental intervention (based on Wesbter-Stratton techniques) seems to have a beneficial effect on children with oppositional defiant disorder and may be particularly suitable for children with irritability, although this needs to be tested further. ${ }^{18}$

\section{Fernanda Valle Krieger, ${ }^{1}$ Argyris Stringaris ${ }^{2}$}

'Department of Psychiatry, University of São Paulo, São Paulo, Brazil

${ }^{2}$ King's College London, Institute of Psychiatry, London, UK

\section{Competing interests None.}

\section{REFERENCES}

1. Perlis RH, Dennehy EB, Miklowitz DJ, et al. Retrospective age at onset of bipolar disorder and outcome during two-year follow-up: results from the STEP-BD study. Bipolar Disord 2009;11:391-400.

2. Leibenluft $\mathbf{E}$. Severe mood dysregulation, irritability, and the diagnostic boundaries of bipolar disorder in youths. Am J Psychiatry 2011;168:129-42.

3. Stringaris A, Santosh P, Leibenluft E, et al. Youth meeting symptom and impairment criteria for mania-like episodes lasting less than four days: an epidemiological enquiry. J Child Psychol Psychiatry 2010;51:31-8.

4. Correll CU, Sheridan EM, DelBello MP. Antipsychotic and mood stabilizer efficacy and tolerability in pediatric and adult patients with bipolar I mania: a comparative analysis of acute, randomized, placebo-controlled trials. Bipolar Disord 2010;12:116-41

5. Findling RL, Youngstrom EA, McNamara NK, et al. Double-blind, randomized placebo-controlled long-term maintenance study of aripiprazole in children with bipolar disorder. J Clin Psychiatry 2012;73:57-63.

6. Miklowitz DJ, Biuckians A, Richards JA. Early-onset bipolar disorder: a family treatment perspective. Dev Psychopathol 2006;18:1247-65.

7. DeFilippis $\mathbf{M}$, Wagner KD. Bipolar depression in children and adolescents. CNS Spectr. Published online: April 2013:1-5. doi:10.1017/S1092852913000217

8. Angst J. Problems in the current concepts and definitions of bipolar disorders. World Psychiatry 2011;10:191-2.

9. Birmaher B, Axelson D, Goldstein B, et al. Four-year longitudinal course of children and adolescents with bipolar spectrum disorders: the Course and Outcome of Bipolar Youth (COBY) study. Am J Psychiatry 2009;166:795-804.

10. Leigh E, Smith P, Milavic G, et al. Mood regulation in youth: research findings and clinical approaches to irritability and short-lived episodes of mania-like symptoms. Curr Opin Psychiary 2012;25:271-6.

11. Olfson M, Crystal S, Huang $\mathrm{C}$, et al. Trends in antipsychotic drug use by very young, privately insured children. J Am Acad Child Adolesc Psychiatry 2010;49:13-23.

12. Stringaris A, Zavos $H$, Leibenluft $\mathrm{E}$, et al. Adolescent irritability: phenotypic associations and genetic links with depressed mood. Am J Psychiatry 2012;169:47-54.

13. Krieger FV, Polanczyk GV, Goodman R, et al. Dimensions of oppositionality in a Brazilian community sample: testing the DSM-5 proposal and etiological links. J Am Acad Child Adolesc Psychiatry 2013;52:389-400.

14. Stringaris A, Baroni A, Haimm C, et al. Pediatric bipolar disorder versus severe mood dysregulation: risk for manic episodes on follow-up. J Am Acad Child Adolesc Psychiatry 2010;49:397-405.

15. Copeland WE, Angold A, Costello EJ, et al. Prevalence, comorbidity, and correlates of DSM-5 proposed disruptive mood dysregulation disorder. Am J Psychiatry 2013;170:173-9.

16. Stringaris A, Goodman R, Ferdinando S, et al. The affective reactivity index: a concise irritability scale for clinical and research settings. J Child Psychol Psychiatry 2012;53:1109-17.

17. Krieger FV, Pheula GF, Coelho $\mathrm{R}$, et al. An open-label trial of risperidone in children and adolescents with severe mood dysregulation. J Child Adolesc Psychopharmacol 2011;21:237-43.

18. Scott S, O'Connor TG. An experimental test of differential susceptibility to parenting among emotionally-dysregulated children in a randomized controlled trial for oppositional behavior. J Child Psychol Psychiatry 2012;53:1184-93. 